\title{
An Advanced IoT-Based Tool for Effective Employee Performance Evaluation in the Banking Sector
}

\author{
Adel Alti ${ }^{1,2 *}$, Ahmed Almuhirat ${ }^{2}$ \\ ${ }^{1}$ LRSD Laboratory, Computer Science Department, Sciences Faculty, Ferhat Abbas University SETIF-1, Sétif 19000, Algeria \\ ${ }^{2}$ Department of Management Information Systems, College of Business \& Economics Qassim University, P.O. Box 6633, \\ Buraidah 51452, KSA
}

Corresponding Author Email: a.alti@qu.edu.sa

https://doi.org/10.18280/isi.260111

Received: 2 November 2020

Accepted: 18 January 2021

\section{Keywords:}

IoT, human resource, smart technology, decision-making, intelligence, performance metric

\begin{abstract}
Nowadays, high-quality service becomes pivotal to ensure higher customer satisfaction for banking sectors. Through developmental methods, it helps them align their employees and resources to meet their strategic objectives. The challenge is that there is no automatic, strong and intelligent way, which helped the bank sectors to assess employee's performance after receiving training while keeping scores. In this paper, we design and implement an effective tool based on IoT for assessing employees' performance by applying the right evaluation metrics. Besides, we aim at determining the necessary information so that the manager has clear strategy that improves performance expectations and keeps it high. We develop also an evaluation model that can take incoming performance data of deployed sensors with work environments, performing data analysis of various employers and determine their performance while customizing the multi-criteria decision-making. The experimental results show that IoT-based tool generates remarkably higher performance than existing tools in the literature for nearly all training programs and all decision-making managers.
\end{abstract}

\section{INTRODUCTION}

Today we are witnessing the big evolution of business organizations in their environment, whether public or private, and in all economic, social, technological, and other fields. With enormous competitiveness in the business world, organizations tend to build collaborations to increase their productivity, improve the efficiency of their business activities, and be well-positioned compared to other competing companies in the market. Therefore, while the customers' demands for quality services are increasing, the real takeaway for companies is that better human skills development increases the company's quality long life. Due to its bulky services, the efficiency and effectiveness of their Human Resource (HR), and the quality-per-deploy model, more and more companies are attracted to automate the assessment of employees' performance after performing a training program. Training is a major method that develops creative skills and an important matter for enterprise leaders. Therefore, effectively monitoring employees with high-efficiency performance is essential to ensuring the success of the bank system. The manager must be capable to exploit real-time HR capacities in strategic business activities and decision making [1].

Without an effective and rigorous performance model in the bank system, most managers do not have the time to ensure better decisions making and a clear vision for the future of the banking sector. Besides, the efforts are being consumed to effectively use the Information and Communication Technologies (ICT) in most of the current assessment process of an employee's job performance. This technology is not fully exploitable, the companies lose quality and consistent assessment to support increasing customer demands. For that reason, an automatic and effective tool to assess the efficiency and effectiveness of HR has become one of the greatest concerns. The main objective is to offer a novel automatic and intelligent strategy to monitor employee behavior and evaluate the employee's performance capabilities after implementing training programs in the banking sector, which assists a manager in better decision making.

Due to the frequent visits and meetings with Qassim Bank office, it becomes clear to researchers that it needs automatic and effective strategies to improve the evaluation of employees at the bank's sector after launching the training program. The bank sector lacks modern strategies that depended on ICT and IoT that help a manager to monitor employees in the bank sector and control many employees to accomplish their activities. With traditional performance appraisal strategies, the manager requires new efforts, and subsequently a significant time delay. Therefore, designing a new performance appraisal model to support the different levels of performance data collection, performance data analysis and intelligent decision-making still require much more effort. The main questions regarding this research study are as follows:

- What is the level of effectiveness and efficiency of the training program in its dimensions (training contents, training requirements, training tactics) for the bank's employees (employee's profile and skills) after receiving it?

- What is the impact of feedback frequency on task performance?

Over the last two decades, various research approaches have 
been proposed for the evaluation of employee appraisal in social and economic domains. These approaches can be divided into three categories: 1) In 360-degree feedbacks approaches [2], opinions leaders were used in evaluation performance 2) In objectives-based approaches, objectives are defined based on standard measures, and employee's profiles are evaluated using these measures [3]; and 3) In statistical approaches, various statistical measurements are used to evaluate an employee's job [4]. In addition to these approaches, multi-criteria IoT-based analysis is incorporated into the evaluation process. To calculate the efficiency and effectiveness scores of employees, standard measures are used.

Many tools have been proposed for assessing employee performance systems for banking sectors. The existing tools have proposed the set of metrics capable of evaluating employees based only on their performances [1,3]. Others discussed the benefits and challenges of deploying logic and physical sensors on the workplace [4-14] but did not examine in detail their impact on employee morale and engagement. Currently, there is no strong and intelligent way to assess and improve employee performance after receiving training while keeping scores and reduced evaluation time. It will be necessary that consider performance data related to smart devices allowing a fast track of performance improvement, continuous performance evaluation of employees to operating as efficiently or effectively as they could. This paper aims to design and implement an advanced IoT-based multi-criteria tool to monitor, analyze, decide and promote the performance of its benefits for business, banking sector, and customers. The main contributions of this work are:

- A multi-criteria decision-making and IoT-based tool are developed for effective and efficient employee performance. The customized evaluation standard metrics have been used to assess employee's efficiency and effectiveness and achieve accurate decisions making.

- Several qualitative and quantitative assessment methods are used to ensure effective decision-making, which can take incoming performance data of employees, ongoing performance needs of various employees and determine their effectiveness and efficiency into banking systems. To the best of our knowledge, this is the first study to include all of these facilities into one single system that aims to evaluate the employee's jobs.

- The evaluation of the results in this study is done using two different methods: Impact of a training program on employee's performance and sensor's frequency to prove that the suggested tool outperforms other existing 360degree feedbacks tools [4].

The remainder of the paper is organized as follows: initially, we discuss the related works in the area of IoT technology in companies in section 2 . In section 3 , the proposed IoT-based tool for monitoring and evaluating the performance of employees is presented. Moreover, section 4 shows a comparison between automatic evaluation tool results, and the traditional evaluation tool results to prove the effectiveness of our proposed approach. Finally, Section 5 concludes the paper, and future research directions are outlined.

\section{RELATED WORKS}

Internet of Things (IoT) is one of the greatest tools that show influences on more modern organizations' performance, which commutate with themselves and other Internet-enabled devices. They helped us to remotely monitor and control the physical world. IoT aims to improve employees' efficiency in their companies with minimum effort. This section presents the review of available tools and IoT-based approaches with their weakness and advantages.

Kiranmai et al. [5] considered IoT as important modern technological transformations that help banks to collect data coming from different sensors and exchange it with other sensors for effectively tracking and analyzing consumer's behavior, providing a practical experience on IoT technologies with high performance, thus making bank achieving their goals. In another study [6], the impact of IoT on the workplace in organizations was researched. This study showed that employee participation levels are necessary for business organizations that use IoT, which had an influential role in this activity. The obtained results showed that IoT has a mediating role in contributing to the digital development of organizations and raising their performance.

According to a recent analysis [7], modern companies are interested in advanced tools and innovative solutions based on information technology that helps to integrate their operations, mechanisms, and employees as one network. IoT web-based tool enables companies to collect, analyze, evaluate, and improve their performance. According to surveys sent to manufacturing and logistics companies to investigate how IoT tools could be used to help solve their problems, companies now have more internet-connected objects that can spread data in real-time thanks to appropriate IoT-based tools and analytical methods. Besides, it showed that the companies that use IoT enjoy a higher level of logistics services, more efficient services with their partners, higher financial market performance, better productivity, and increased economic sustainability and competitiveness. However, existing IoTbased not able to support customized decision-making. To provide such support, we will present in the next section the IoT-based multi-criteria tool that enables the automatic and customized evaluation of employee's efficiency and effectiveness. Authors in the ref. [8] have mentioned that IoT may offer many benefits for organizations. According to an analysis conducted on how asset management is used to evaluate the benefits and risks, IoT provides organizations with more benefits after dealing with their risks. As a result, IoT is expected to reach the organization's capabilities by making organizational and operational changes that help in achieving the organization's goals. However, it still developing an advanced IoT-based framework that can provide agile and automatic techniques while keeping continuous user's evolution skills and automated employee management and evaluation. Shafik et al. [9] have adopted the best technology to remote control over IoT applications, leading to more effective use of data collection in the industrial organization and better solutions to proactively solve or prevent many issues. The results of this study showed that IoT could provide reliable and efficient systems and provide highquality products. Recently Shafik et $a l$. have integrated IoT to mobile bank applications for ensuring security, congestion control, flow control, and energy efficiency [10]. Once the performance data is collected from wearable devices, and for each employee, proof of validation and integrity is retrieved from the central database and added to modern technology. Other research works have integrated IoT-connected employees for IT departments [11-14], but none of them are focusing on employee's performance after receiving a training through automatic, strong and intelligent way. 


\section{IOT-BASED EMPLOYEE PERFORMANCE EVALUATION TOOL}

The proposed system considers a general banking office and IoT Devices in which the IoT devices track employees to provide the manager with the requested performance data. The main aim is to increase the employee's efficiency and effectiveness after receiving a training program. Particularly, the goals that are to be achieved are as follows:

- Efficiency: The proposed performance monitoring and evaluation method should help leaders to monitor employees' efficiency in terms of number of achieved tasks and accomplished time.

- Effectiveness: The proposed performance monitoring and evaluation method should help leaders to monitor employees without the intervention of users.

\subsection{General architecture}

The proposed system involves three modules that collaborated-together to accomplish the system goal. The components of the proposed system are the employer, the server, and the manager. The system architecture of the proposed model with the three modules is shown in Figure 1 and described as follows:

- $\quad$ Employer module: consists of the employer profile and IoT module. The IoT module collects information about the training program and its dimensions (contents, tactics, and requirements) and information about the employee's profile and its static and dynamic dimensions (gender, qualification levels, skills, flexibility). The IoT module consists of various sensors (camera, motion sensor, and task report sensors) and mobile devices such as smartphones, smart-TV as well as PC. These devices measure the key performance data (accomplished tasks, treatment time) and send it directly to the server manager. This process is carried out semi-automatically with the need to interact with an employer to accomplish some accomplished tasks.

Performance server module: the server is responsible for providing report analysis for a manager. The server receives performance data from the employer module and produces global performance reports. It evaluates employee performance in terms of efficiency and effectiveness at each bank service. The goal is to provide the directed managers with requests performance and analysis reports for decision-making service.

- $\quad$ Manager module: this module enables a manager at trusted bank offices to monitor and track the employer's performance data and acts as a decision-making server. This enables a manager to predict any unperformed activity and can assign training programs to prevent any emergency case from the training programs repository. This module is synchronized with the server to receive updates of the employer's performance analysis reports. A customized weighted criteria mechanism is used to evaluate received performance data and deliver it to the monitoring dashboard.

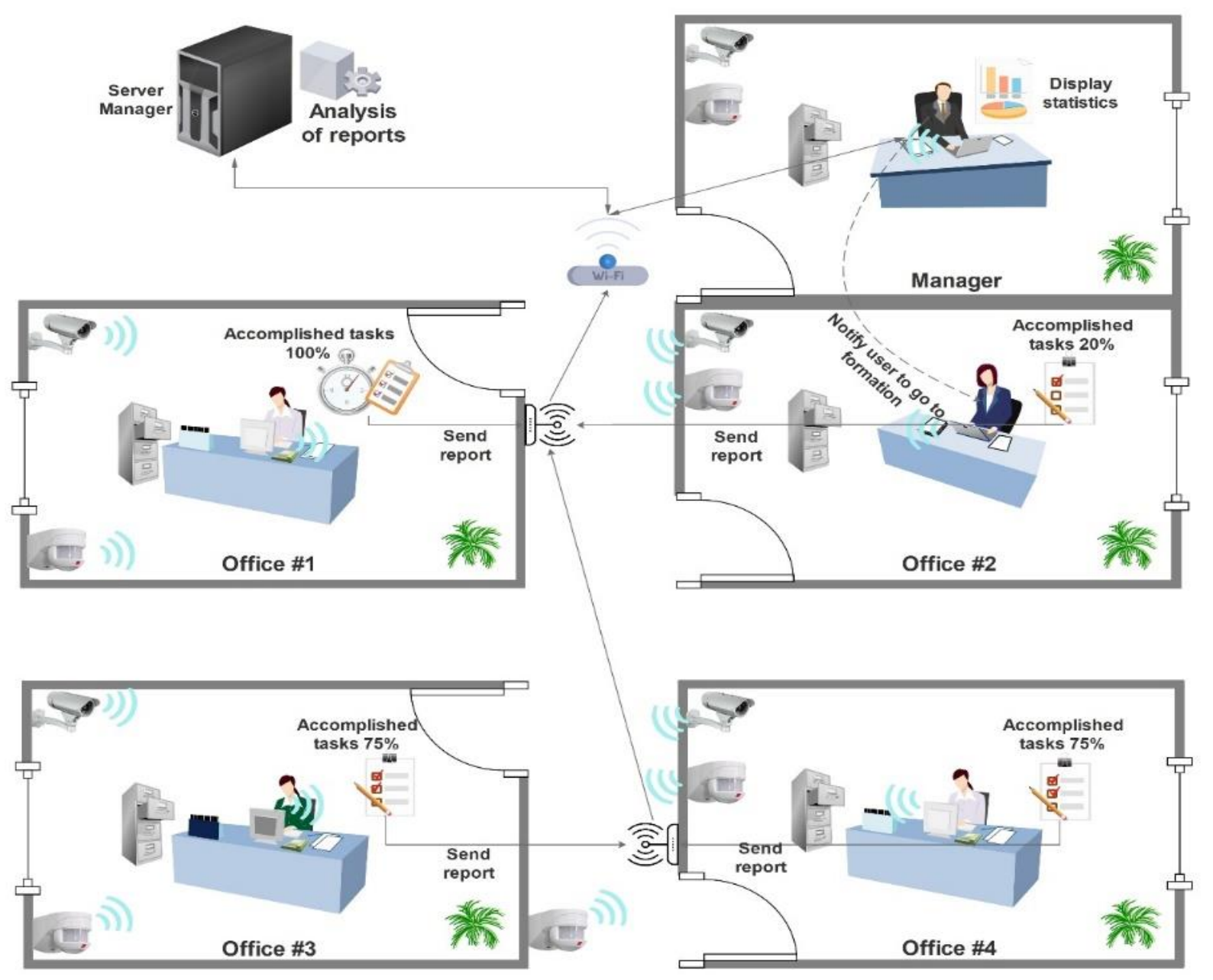

Figure 1. Comparative study of four smart domains 


\subsection{Functional model}

The complete sequence diagram is described in Figure 2. When an employee configures his session, he provides performance information. Some information are automatically collected from IoT devices of the employer module, and others are specific to an employee. IoT devices capturing raw performance data (i.e. accomplished tasks, deadlines, etc.) and send it to the server manager.

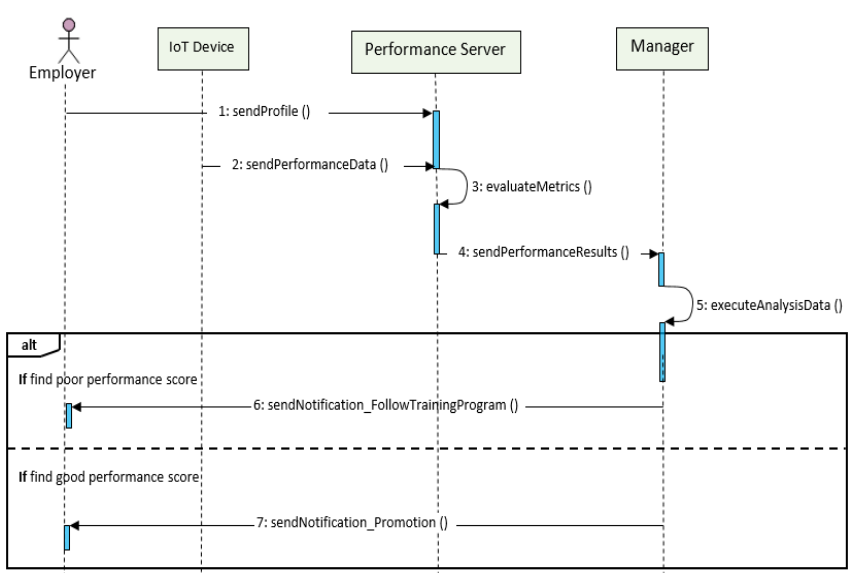

Figure 2. Sequence diagram of our system

We distinguish three sensor types: smart camera sensor, motion sensor, and task report sensor. The camera sensor is involved in this study to monitor the activity rate (i.e. checks emails). The motion sensor produces true value for the human presence or false instead. Task report sensor is a logical sensor used to measure the employer accomplished tasks and employer's behavior (i.e. what the employer is performing on his post/operating time/the time of using the mouse or keyboard). The employer module establishes a connection to the server database over a Wi-Fi connection and sends it the performance data. Since the server receives the performance data of the employer, it runs the Performance Evaluator to produce analysis reports according to manager-defined weights and performance standards. These analysis reports are stored in the database and the manager is notified of new analysis reports. The manager login via a web interface to be authenticated. They are directed to the monitoring dashboard and display statistical analysis reports of the employer.

- If there is a poor performance score, he sends a notification to the employee ask him to follow/restart another training program for increasing the employee's commitment and confidence.

- If there is a good performance score, he sends a notification to the employee about the promotion decision for increasing the employee

\subsection{Performance standards and customized evaluation mechanism}

The evaluation mechanism depends on two main criteria: efficiency and effectiveness. Efficiency and effectiveness are the most widely used criteria these days in assessing and measuring the employee's performance at different levels. However, achieving both may not always be possible due to potential tradeoff between them. A suitable evaluation of performance standards can be obtained by customizing and analyzing these criteria (i.e. efficiency and effectiveness) making a better evaluation of employee performance. The primary use of such evaluation is allowing us to explore the consequences of different decisions about the training program. For example, the manager may prefer to use a few services for reducing the time to reach the target training goals. Alternatively, if time is not a constraint, and effectiveness is an important factor under constrained resources, the manager may decide to stretch the adaptation out over a larger number of performed tasks. To ensure customized evaluation, two key criteria were evaluated for each employee and then recorded at least one week later. Efficiency and effectiveness were assessed using serval standard parameters (Table 1). Currently, each quality parameter is valued by the manager according to its scores. It is an integer number that indicates the maximum degree of scores (10 for excellent, 8 for very good, 6 for good, 4 for medium, and 2 for low). In our model, the quality parameters are associated with performance percentage specification such as:

1. "Excellent" when an employee achieves 80\% - 100\%

2. "Very Good" when an employee achieves $60 \%$ $79 \%$

3. "Good" when an employee achieves 40\% - 59\%

4. "Medium" when an employee achieves 20\% - 39\%

5. "Low" when an employee achieves less than $20 \%$.

The efficiency of each employee is evaluated in terms of:

6. Number of accomplished tasks.

7. Quality of results/tasks.

8. Number of errors.

9. Customer's satisfaction degree.

10. Number of technical skills.

11. Number of strategies.

Table 1. Performance criteria evaluations with its scores.

\begin{tabular}{|c|c|c|c|c|c|c|}
\hline Criteria & Metrics & Excellent (10 & Very Good (8) & $\operatorname{Good}(6)$ & Medium (4) & $\overline{B a d}(2)$ \\
\hline Efficiency $\left(W_{1}\right)$ & \begin{tabular}{|c|} 
Quality of results/tasks \\
Number of errors \\
Customers satisfaction degree \\
Number of technical skills \\
Number of strategies \\
\end{tabular} & & & & & \\
\hline Effectiveness $\left(\mathbf{W}_{2}\right)$ & \begin{tabular}{|c|} 
Size of work \\
Finished tasks \\
Scheduled appointments \\
Planning of work \\
Total treatment time \\
\end{tabular} & & & & & \\
\hline \multicolumn{2}{|c|}{ Evaluations (\%) } & $80-100$ & $60-79$ & $40-59$ & $20-49$ & $<20$ \\
\hline
\end{tabular}


The effectiveness of each employee is evaluated in terms of:

1. Size of work.

2. Number of finishing tasks.

3. Number of scheduled appointments.

4. Number of unscheduled emergency appointments.

5. Total treatment time.

We define the evaluation function that measures the performance of the employee as follows:

$$
\text { Score }=W_{1} \times \text { efficincy }+W_{2} \times \text { effectiveness }
$$

where, $\mathrm{W}_{1}$ and $\mathrm{W}_{2}$ are coefficients that allow the manager to specify the importance of each criterion using GUI. All these weights are set between 0 and 1 . One manager may specify in advance that the time is not a constraint and that he prefers high-quality products, while another one may want fast time with an acceptable quality of work.

\section{IMPLEMENTATION AND EVALUATION RESULTS}

Our prototype is implemented with NetBeans 7.2. The IoTbased decision-making process of employee's performance is the main part of our framework. The prototype is able to track and evaluate employee's performance under different jobs complexities. The performance data are frequently captured and sensed (every 300 milliseconds) by different sensors. It allows as much capturing of incoming performance data as possible in a parallel way to process them in a parallel way, which improves the performance system and reduces the response time. There are various RFID sensors. Each sensor serves to monitor information such as a smart camera to monitor the employer's activity (e.g., number of achieved tasks and accomplished time or checks emails), a smartwatch to monitor the user's locations, and other sensors to detect other information such as planned tasks.

We present two experiments on smart banking offices. The first one consists of evaluating the impact of training programs on employee performance. The second one deals with the effectiveness of using an IoT-based performance system and its comparison performance with a traditional 360-degree feedbacks performance system [2]. We will validate the effectiveness of our IoT-based employee performance system and prove that it can provide the manager with a first guide to predict the employee's performance by considering a connected smart bank.

\subsection{The impact of training program on employee's efficiency and effectiveness}

In this experiment, a set of 50 employees is used in the proposed IoT-based performance evaluation system including 30 males $(60 \%)$ and 20 females $(40 \%)$ aged $22-50$ years. Besides, the task deadline is set to 30 minutes, 1 hour, 2 hours according to easy, medium, and complexity levels.

The manager assigns to each employee 10 tasks with different complexity levels after acquiring training program, which is enough to evaluate efficient employee effort. We use a set of 70 sensors related to smart bank offices as described in Section 3.2 (8-surveillance camera, 50 employee presence detectors, and 50 task report sensors). Sensors performance data was simulated automatically using JSON files between 8:00 pm and 02:00 pm.
4.1.1 The impact of training program on employee's efficiency We evaluated the impact of training program on the efficiency scores of each employee in terms of quality of tasks results, number of errors, customer satisfaction level, number of technical skills, and number of strategies for one week after training program. Evaluation results are shown in Figure 3. The quality of task results and number of errors are different between employees either male or female. Since the number of errors and the quality of tasks results depends directly on the strategies of training program (i.e. technical skills). The number of errors has improved because the training program is selected according to the exact event triggering errors, which allows, in most cases, to select the correct training program. Thanks to the real-time employee's tracking data offered by IoT-enabled multimedia devices (i.e. camera sensor, logical sensors), managers receive these useful data they allow them to track how employees perform works and improve strategies of work by selecting the right training and allow the employees to perform their jobs faster, easier, while enjoying greater added value. This will lead to customer satisfaction as their goals are achieved.

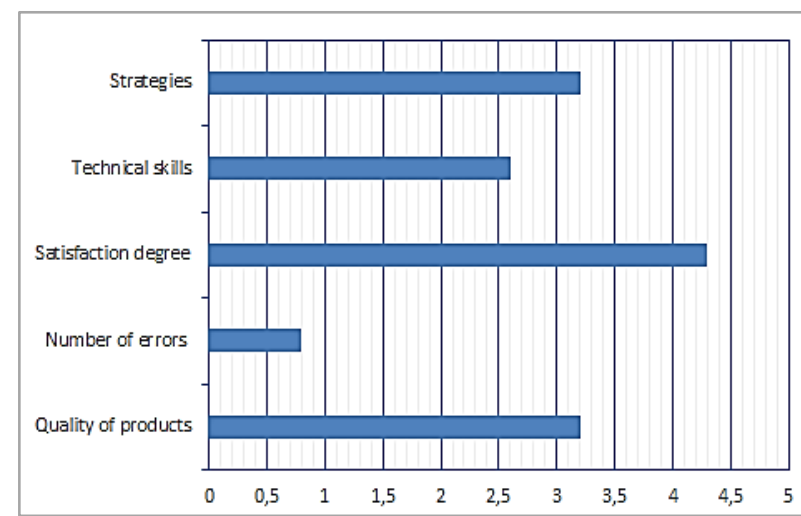

Figure 3. Weekly average efficiency scores

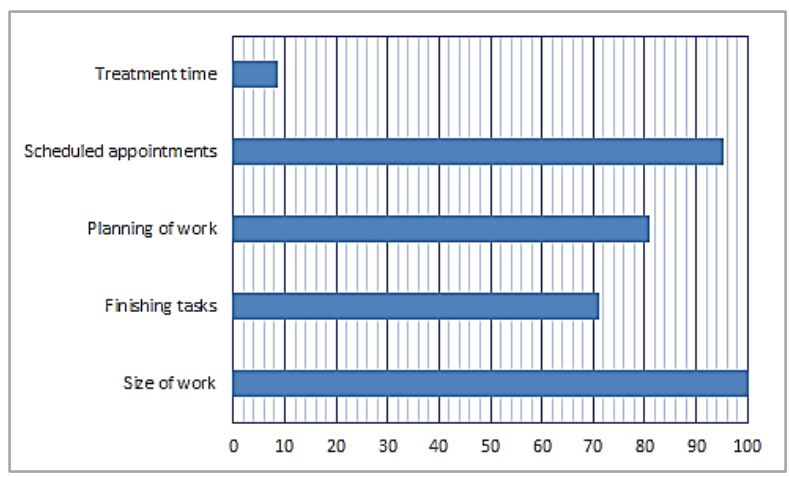

Figure 4. Weekly average effectiveness scores

4.1.2 The impact of training program on employee's effectiveness

In this experiment, we have evaluated the impact of training program on the effectiveness scores of each employee in terms of size of work, finished tasks, scheduled appointments, planning of work, and treatment time for one week after a training program. The treatment time was significantly shorter for the Insignia patients, with about seven days' appointments on average as shown in Figure 4. Furthermore, the complexity level of task, quality of training program cause the decrease in the time consumed by performed tasks. We can notice that in most cases, managers take full advantage of IoT to collect 
much more performance and make more right training decisions. In this context, exploiting all available sensors to detect space utilization while simultaneously tracking employee's performance can be great steps on the strategy to increase employee engagement and productivity. This exploitation resulted in an overall 15 percent increase in productivity among the bank's employees compared to the traditional approaches.

\subsection{The impact of sensor's feedback on employees evaluation time}

We have compared the impact of varying sensor's frequency on the employee's evaluation time on the IoT-based approach and traditional approach without IoT. Table 2 shows the employee's evaluation comparison of the proposed approach vs traditional approach. As shown in Table 2 at $400 \mathrm{~ms}$ sampling frequency, results show that the proposed approach has an employee's evaluation time of $28 \mathrm{~ms}$. On the other part, the traditional approach has an employee evaluation time of $610 \mathrm{~ms}$. The proposed approach outperforms the traditional framework, which yields faster evaluation time across all varied sensor frequency employing.

Table 2. Average evaluation time comparison of the proposed approach vs traditional approach

\begin{tabular}{ccc}
\hline Frequency (ms) & Traditional approach & Proposed approach \\
\hline 100 & $610 \mathrm{~ms}$ & $28 \mathrm{~ms}$ \\
200 & $600 \mathrm{~ms}$ & $25 \mathrm{~ms}$ \\
300 & $600 \mathrm{~ms}$ & $16.5 \mathrm{~ms}$ \\
400 & $600 \mathrm{~ms}$ & $16.1 \mathrm{~ms}$ \\
500 & $530 \mathrm{~ms}$ & $12 \mathrm{~s}$ \\
\hline
\end{tabular}

\section{CONCLUSIONS AND FUTURE WORKS}

This paper presented an advanced IoT performance employee evaluation tool to help companies achieve automated and enhanced employee performance. It is based on multi-criteria and IoT technology for monitoring and evaluating employee's jobs. It is defined through customized efficiency and effectiveness criteria. We take into account a large number of performance data on the fly. Thus, we automatically and easily provide increased support for decision-makers in companies. Several experiments were conducted using a real-life motivating case study. Experimental results have shown that the proposed approach provides better efficiency while ensuring fast evaluation time. Comparison with the traditional tool shows that the proposed IoT customized approach is more efficient and improves the performance of employees within the organization. Moreover, it provides good support for managers to monitor their decisions and access them in an effective manner. Future work can focus on the flexibility and security-related issues to ensure a complete evaluation of employees in the banking sector.

\section{REFERENCES}

[1] Bini, L., Dainelli, F., Giunta, F. (2016). Business model disclosure in the strategic report. Journal of Intellectual Capital, 17(1): 83-102. http://dx.doi.org/10.1108/JIC-09-
2015-0076

[2] Kanaslan, E.K., Iyem, C. (2016). Is 360-degree feedback appraisal an effective way of performance evaluation. International Journal of Academic Research in Business and Social Sciences, 172-182. http://dx.doi.org/10.6007/IJARBSS/v6-i5/2124

[3] Islami, X., Mulolli, E., Mustafa, N. (2018). Using management by objectives as a performance appraisal tool for employee satisfaction. Future Business Journal, 4(1): 94-108. http://dx.doi.org/10.1016/j.fbj.2018.01.001

[4] Bini, M., Carpita, M., Posa, D., Sarnacchiaro, P. (2019). Socio-economic indicators for performance evaluation and quality assessment: Statistical methods and applications. Social Indicators Research, 146: 1-5. http://dx.doi.org/10.1007/s11205-019-02098-9

[5] Kiranmai, S., Sridharan A. (2017). Internet of thing (IOT)- underpinning the banking. International Journal of Advanced Research in science and Engineering, 1(6).

[6] Nagy, J., Oláh, J., Erdei, E., Máté, D., Popp, J. (2018). The role and impact of Industry 4.0 and the internet of things on the business strategy of the value chain-the case of Hungary. Sustainability, 10(10): 3491.http://dx.doi.org/10.3390/su10103491

[7] Brous, P., Janssen, M., Herder, P. (2020). The dual effects of the Internet of Things (IoT): A systematic review of the benefits and risks of IoT adoption by organizations. International Journal of Information Management, 51 : 101952. https://doi.org/10.1016/j.ijinfomgt.2019.05.008

[8] Salhaoui, M., Guerrero-González, A., Arioua, M., Ortiz, F.J., El Oualkadi, A., Torregrosa, C.L. (2019). Smart industrial IoT monitoring and control system based on UAV and cloud computing applied to a concrete plant. Sensors, $19(15)$ : 3316. http://dx.doi.org/10.3390/s19153316

[9] Shafik, W., Matinkhah, M., Asadi, M., Ahmadi, Z., Hadiyan, Z. (2020). A study on internet of things performance evaluation. Journal of Communications Technology, Electronics and Computer Science, 28: 1-19. http://dx.doi.org/10.22385/jctecs.v28i0.303

[10] Ramamurthy, A., Jain, P. (2017). The internet of things in the power sector opportunities in Asia and the Pacific. http://hdl.handle.net/11540/7821.

[11] da Cruz, M.A., Rodrigues, J.J., Sangaiah, A.K., AlMuhtadi, J., Korotaev, V. (2018). Performance evaluation of IoT middleware. Journal of Network and Computer Applications, 109: 53-65. http://dx.doi.org/10.1016/j.jnca.2018.02.013

[12] Nižetić, S., Šolić, P., González-de, D., Patrono, L. (2020) Internet of Things (IoT): Opportunities, issues and challenges towards a smart and sustainable future. Journal of Cleaner Production, 274: 122877. http://dx.doi.org/10.1016/j.jclepro.2020.122877

[13] Ammirato, S., Sofo, F., Felicetti, A. M., Raso, C. (2019). A methodology to support the adoption of IoT innovation and its application to the Italian bank branch security. context. European Journal of Innovation Management, 22(1): 146-174. http://dx.doi.org/10.1108/EJIM-032018-0058

[14] Dhir, K., Chhabra, A. (2019). Automated employee evaluation using fuzzy and neural network synergism through IoT assistance. Personal and Ubiquitous Computing, 23(1): 43-52. https://doi.org/10.1007/s00779-018-1186-6 